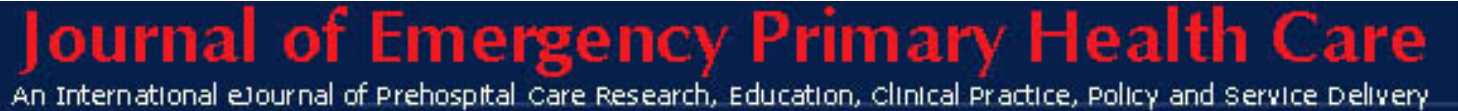

ISSN 1447-4999

POLICY AND SERVICE DELIVERY

Article 990184

Rural and Frontier Emergency Medical Services: Agenda for the Future. National Rural Health Association, 2004. Kansas City. McGinnis, Kevin K.

- An Italian and European Perspective

by Assunta De Luca, Marica Ferri and Gabriella Guasticchi

Public Health Agency of Lazio region, Italy

\title{
The European Perspective
}

Emergency Medical Services in Europe evolved during military conflicts from the need to transport a patient from the battle camp to a physician who could provide definitive care. ${ }^{1}$ Over time, individual countries developed systems that best met the needs of that community, based on culture, local welfare state approaches and relevant laws. Nonetheless, availability of health care resources and finance had had an impact on the development of such systems.

Starting from 1960s, the most evolved systems recognized the role of transport on patient outcomes. As a result, European systems now differ substantially and therefore cannot be directly compared with each other. Currently, some systems are based mainly on the provision of pre-hospital care by non-physicians (emergency medical technicians and paramedics), while others are based on the central role of emergency physicians. As a consequence some EMS systems concentrate interventions on the scene of the emergency, whereas others focus on minimisation of transport time.

Some Basic Life Support (BLS) is provided by volunteers, and full Advanced Life Support (ALS) relies on emergency physicians or ALS paramedics. These differences have a significant impact on resource utilisation (e.g. hospital admission rates, length of stay etc.) and obviously have an effect on health outcomes.

Current effort ${ }^{1}$ is being undertaken by 13 European countries to identify common indicators and monitor the different emergency health strategies, in an attempt to "reduce" differences in the European emergency systems - at least in terms of data collection and storage, and consequently in terms of performance comparability.

\section{The Italian perspective}

Italy belongs to south of Europe where the emergency system reflects a peculiar history and social development. The health system is generalist and is aimed at providing all citizens with a complete and free access to health services.

The Italian health system is delegated to the 21 regional authorities and the national Ministry is responsible for determining Essential Levels of Assistance (described in the triennial Plan, published by the Ministry of Health) and to grant the provision of free care and assistance to 
everyone, regardless of whether he or she is a registered citizen or a non-registered migrant. No barriers based on any kind of criteria should interfere with access to health, as is stated in the Constitution, and which grants free health interventions for poor people.

Due to historical and economical reasons, Italy's regions differ substantially in terms of availability and quality of services. Significant effort is therefore devoted to the homogenisation of services among the regions. For this to occur, information systems to monitor and assess the services would need to be implemented to achieve a satisfactory level of comparison.

The Emergency System in Italy is activated by dialling '118' (http://www.118italia.net) 24/7. The constitution of this service and the improvement of it, has led to the side effect of rising requests for interventions for health conditions which should not be regarded as an emergency.

In fact, the presence of long waiting lists for many interventions in the health services, and the promptness of ' 118 ' interventions has constituted an incentive for people to over demand emergency interventions.

To rationalize resources and coordinate interventions more effectively, it is essential to rely on the dissemination of good quality information regarding current practice in the different regions. To satisfy this need, since 2004, the Italian Ministry of Health has supported a project for the classification of interventions, in which emergency interventions are grouped and classified in a similar way to the Disease Related Group (DRG). Common criteria for the collection and storage of information are adopted, and guidelines for the harmonization of the different structures involved in the EMS, are implemented.

The Project is called "Progetto Mattone Pronto Soccorso e Sistema 118", where "mattone" means "brick" and symbolizes the building up of a system developed from the integration of several structures ${ }^{2}$.

The challenge is to create a network which is able to coordinate the service without trespassing on the autonomy of the regions. For this reason, the leadership of the project has been committed to one region, the Lazio region, where the capital, Rome, is located.

Moreover the leadership was given to Lazio as it is the only region with a health information system based on data currently collected in all hospital emergency departments (Information System of Emergency Services - SIES) through a computerized clinical chart. Given that the SIES was activated in 1999, the long experience of Lazio region was therefore considered a good starting point for the implementation of the national project.

\section{Future perspective}

In the development of the Italian project, priority has been given to researching similar international and local issues in an effort to identify methods and solutions which will assist in improving our current problem.

In this sense reading the Rural/Frontier EMS Agenda for the Future by the National Rural Health Association was very relevant as it provided an insight into similar issues which are ocurring in other parts of the world, as they also strive to overcome the problems of offering an efficient emergency service to large areas where communications are challenged. 
The Rural/Frontier EMS Agenda for the Future is a good example of strong collaboration between all rural health associations and another important authority, the US Department of Transportation, to identify needs and criticisms of rural EMS, and provide suggestions to local policy makers on how to overcome problems with existing resources and plan more effectively for the future.

Due to the immensity and diversity of the territory, the American experience provides an essential contribution to other countries in the world in terms of planning an integrated emergency health service. The elements of common vision of the rural/frontier emergency medical service (EMS) system of the future are to: assure an appropriate rapid response to each emergency; and, provide the community with prevention, evaluation, care, triage, referral and advice. Its point of strength is identified in the consolidated collaboration between volunteer and health personnel at all levels.

As the EMS is considered an important component of the rural system of care, the Agenda shows how the development of an integrated emergency medical safety net is possible. To achieve a common vision, health professionals, stakeholders, national and local policy makers must work together to solve issues such as human resources and infrastructure, development of non-traditional education methods vs traditional methods, the role of the volunteer, adequacy of communications infrastructure, quality of data collection, development of an evaluation system of EMS activities, community campaign to inform how and when to use the EMS system and, implementation of a national on-line and off-line medical oversight network. All of these issues are commonly found in Europe as they are in Italy, not only in rural areas, but also in urban areas. For this reason, all projects currently underway or about to be embarked upon, must strive to find better solutions to overcome the existing problems.

Other areas which are considered as equally important in Italy are, volunteerism, and the implementation of a medical oversight network. Both are current hot topics, seen as a necessity in areas where resources are lacking, and where the availability to exchange experiences and medical oversight face-to-face is limited. Communication and collaboration of rural and frontier EMS providers is more crucial today than ever before, as the increasing threat of mass emergencies or terrorist attacks have become a reality.

In Europe, as in America, the biggest task is in how to motivate the policy makers to put into practice, those quality improvements, which are clearly outlined in the Agenda for the Future, into our EMS systems of the future.

\section{References:}

1 Krafft T et al. European Emergency Data project (EED Project). Eur.J.Pub.Health 2003; 13 (3 supp.):85-90.

2 Palumbo F. Il programma "Mattoni del SSN”. Monitor 2005; Anno IV , 13. www.assr.it. 